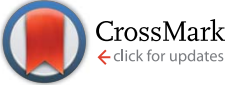

Cite this: RSC Adv., 2017, 7, 9562

\title{
Self-assembled hybrid hydrogels based on an amphipathic low molecular weight peptide derivative and a water-soluble poly(para- phenylene vinylene) $\uparrow$
}

\author{
Jenifer Rubio-Magnieto, ${ }^{\text {a }}$ Marta Tena-Solsona,,$^{\text {ab }}$ Beatriu Escuder ${ }^{* b}$ \\ and Mathieu Surin ${ }^{\mathrm{a}}$
}

Received 4th November 2016 Accepted 24th January 2017

DOI: 10.1039/c6ra26327e

rsc.li/rsc-advances
The self-assembly of an amphipathic low molecular weight peptide and a water-soluble $\pi$-conjugated polyelectrolyte is studied in order to form hybrid hydrogel materials with synergistic properties. The self-assembly of the complex yields a network of one-dimensional fibrils, with enhanced fluorescence of the $\pi$-conjugated polyelectrolyte in the hydrogel form. These hybrid hydrogels are also tested to study the kinetics of release of a molecular dye.
The fabrication of soft materials like hydrogels is an emergent approach for potential applications in different areas such as catalysis, sensing, cosmetics, and biomedicine. In particular, hydrogels are very appealing as biomaterials for drug delivery, tissue engineering, cell culture, and antimicrobial surfaces. ${ }^{1-4}$ Molecular hydrogels are formed by supramolecular selfassembly of low molecular weight gelators (LMWGs), which are based on small molecular building blocks, such as peptides. ${ }^{5,6}$ Recently, the combination of these LMWGs with polymers has appeared as an attractive approach in the field of gels, where the addition of a non-gelling polymer to the low molecular weight gelator has an effect on the LMWG network. ${ }^{7}$ One of the first examples of this combination LMWG/polymer was given by Hanabusa and co-workers, who showed that the addition of different polymers such as poly( $N$-vinylpyrrolidone) or poly(ethylene glycol) enhanced the strength of the gel, which was evaluated by measuring the power needed to sink a cylindrical bar into the gel material. ${ }^{8}$ Indeed, the self-assembly of a gelator and a non-gelling additive through supramolecular interactions represents an appealing strategy to bring new functionalities to a gel. ${ }^{9}$ The polymer can also exhibit modified (enhanced) properties due to its incorporation presence within the gel network. ${ }^{1,7}$

${ }^{a}$ Laboratory for Chemistry of Novel Materials, Center for Innovation in Materials and Polymers, University of Mons - UMONS, 20 Place du Parc, B-7000 Mons, Belgium. E-mail: jenifer.rubiomagnieto@umons.ac.be; Tel: +32 65373865

${ }^{b}$ Departament de Química Inorgànica i Orgànica, Universitat Jaume I, Avenida Sos Baynat s/n, 12071 Castelló, Spain.E-mail: escuder@uji.es

$\dagger$ Electronic supplementary information (ESI) available: Synthesis and preparation of the gels and mixtures, additional spectra and microscopy images. See DOI: 10.1039/c6ra26327e

+ Present address: Fakultät für Chemie, Technische Universität München, Lichtenbergstraße 4, 85748 Garching, Germany.
In this context, Smith et al. have worked on the fabrication of functional materials by combining polymers and selfassembling gelators, observing that the formed hybrid structure possesses modified behaviour which could be used to generate 'nano-imprinted' materials. ${ }^{10}$ Recently, the same group also obtained hybrid hydrogels based on a low molecular weight gelator and a natural polymer gelator (agarose), in which the integrity of the gel is retained thanks to the greater resilience of the agarose network. ${ }^{11}$ Ulijn et al. obtained a controlled release of proteins by using enzyme-compatible poly(ethylene glycol acrylamide) particles, which were functionalised with peptide actuators in order to form enzyme-responsive hydrogel particles. ${ }^{12}$

In this paper, we report on the design of supramolecular gels via self-assembly of a low molecular weight peptide-based gelator (named ZFKFK, see Chart 1a) and a water-soluble $\pi$ conjugated polyelectrolyte, poly[5-methoxy-2-(3-sulfopropoxy)1,4-phenylenevinylene] potassium salt (PPV, Chart 1b). For the molecular hydrogel, we selected a tetrapeptide LMWG that combines hydrophobic aromatic phenylalanine and hydrophilic lysine residues. This peptide derivative has been shown to
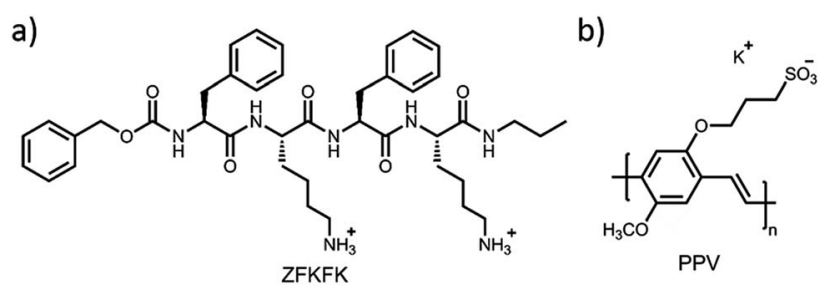

Chart 1 Chemical structures of (a) lysine-based compound, ZFKFK and (b) poly[5-methoxy-2-(3-sulfopropoxy)-1,4-phenylenevinylene] potassium salt (PPV). 
form hydrogels at relatively low concentrations (a few $\mathrm{mM}$ ), depending of the $\mathrm{pH}$ as well as co-assembled gels in combination with $-\mathrm{COOH}$ containing gelators at neutral $\mathrm{pH}^{13}$ We selected a $\pi$-conjugated polyelectrolyte, poly[5-methoxy-2-(3sulfopropoxy)-1,4-phenylenevinylene] potassium salt (PPV), for its good solubility in water and its high fluorescence quantum yield, possibly providing attractive fluorescence properties to the gels. ${ }^{14-16}$ This particular polymer was selected because of it can interact via ionic self-assembly between the polymer anionic side-groups and the peptide cationic lysine amino acids, in addition to $\pi-\pi$ interactions between the polymer backbone and the peptide phenylalanine side-groups. By mixing the LMWG compound (in non-gel or gel state) in aqueous solution with the water-soluble fluorescent polymer (PPV), we aim at fabricating polymer-peptide supramolecular hybrid hydrogels with synergistic properties. The solutions and gels were thoroughly studied by UV-Vis absorption, fluorescence, circular dichroism (CD), atomic force microscopy (AFM), and transmission electron microscopy (TEM).

The ZFKFK compound was dissolved in aqueous TRIS buffer (pH 7.4) at three different concentrations $(0.6 \mathrm{mM}, 6 \mathrm{mM}$, and $15 \mathrm{mM}$ ), it is to say two concentrations below $(0.6 \mathrm{mM}$ and 6 $\mathrm{mM})$ and one above $(15 \mathrm{mM})$ the minimum gel concentration (MGC) of pure ZFKFK. The MGC was determined at around $13 \mathrm{mM}$ at a pH of 7.4 using the test tube inversion method (see Fig. 1a and b). To obtain mixed solutions and mixed hydrogels of ZFKFK : PPV, solutions of ZFKFK were gently heated until complete dissolution was achieved. A small and exact volume of a)

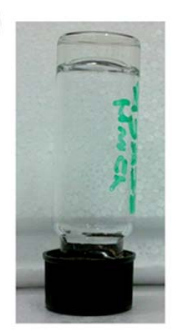

b)

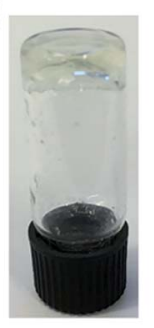

c)

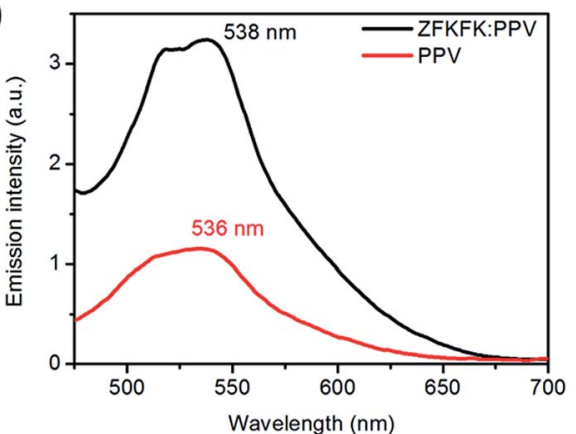

d)

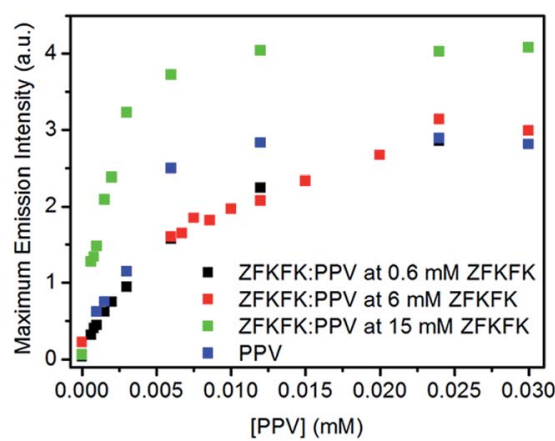

Fig. 1 Photographs of (a) pure ZFKFK and (b) mixed hydrogel; (c) emission spectra (at $\lambda_{\text {exc }}=451 \mathrm{~nm}$ ) of PPV $(3 \mu \mathrm{M})$ pure and when gelled with ZFKFK; (d) emission intensity at $538 \mathrm{~nm}\left(\lambda_{\text {exc }}=451 \mathrm{~nm}\right)$ of PPV into a solution $(0.6 \mathrm{mM}$ and $6 \mathrm{mM}) / \mathrm{gel}(15 \mathrm{mM})$ of the peptide at different molar ratio and PPV at different concentrations. aqueous solution of PPV (0.22 $\mathrm{mM})$ was added over warm solutions of peptide and the mixtures were left to equilibrate at room temperature in order to form the hybrid solutions with the presence of $0.6 \mathrm{mM}$ and $6 \mathrm{mM}$ of peptide and the hybrid hydrogel with the $15 \mathrm{mM}$ of peptide. UV-Vis spectra of the ZFKFK : PPV mixtures show red-shifted spectra compared to the pure PPV (see Fig. S1†). This may indicate a more conjugated PPV backbone upon ionic self-assembly with ZFKFK, especially for the mixture in which the ZFKFK is in its gel state, showing the larger red-shift of the lowest-energy peak (red-shift around $23 \mathrm{~nm}$ ).

Fluorescence spectroscopy was carried out with ZFKFK below its MGC ( $0.6 \mathrm{mM}$, non-gelated form) mixed with different concentrations in PPV (see Fig. S2 $\dagger$ ). The emission spectra of the PPV: ZFKFK complex shows a red-shifted emission maximum compared to the pure PPV, further indicating an interaction between both components. The emission intensity is lower than the one of the pure PPV. For mixtures with a higher peptide concentration (around $6 \mathrm{mM}$, non-gelated form), the fluorescence experiments show rather identical results than using the $0.6 \mathrm{mM}$ ZFKFK in the mixture: the fluorescent emission intensity of pure PPV and PPV : ZFKFK complex is similar (Fig. S2c and $d \dagger$ ).

Very interestingly, when the tetrapeptide ZFKFK in gel state (15 $\mathrm{mM}$ ) is mixed with the $\mathbf{P P V}$, the mixture presents a significant enhancement of the fluorescence emission, see Fig. 1c. Fig. $1 \mathrm{~d}$ shows the intensity of emission maxima as a function of the amount of added PPV into a solution of peptide, at the three different concentrations. We observed that the enhancement of fluorescence occurs only when the ZFKFK : PPV complex is gelled (at $15 \mathrm{mM}$ ). In particular, at low concentrations of PPV (below $6 \mu \mathrm{M}$ ), the fluorescence was around $60 \%$ higher than the emission of pure PPV, whereas at higher concentrations in PPV, the enhancement is around $30 \%$. This is in contrast to a previous report that showed that the addition of a cationic surfactant to an anionic PPV quenched the fluorescence of the polymer. ${ }^{17}$ This points out that the self-assembled structure of the gel plays an important role and influences the conformation/aggregation of PPV, and therefore its emission properties. Similarly, Kuang et al. pointed out an enhancement of chiroptical activities in different nanoparticles (NPs) by using different templates, ${ }^{18,19}$ for instance a luminescent enhancement was observed for gold nanorod (NR)-UCNP tetramers functionalized with complementary DNA by tuning the distance between the upconversion nanoparticles (UCNPs) and the gold nanorods (NRs). ${ }^{20}$

The complexation of PPV $(0.012 \mathrm{mM})$ with the tetrapeptide derivative was further studied by means of circular dichroism (CD) spectroscopy, at the three different concentrations described above. As reported earlier, the reorganization of the peptide chromophores during the self-assembly/aggregation process can lead to important changes in the CD signals when going from solution to the gel state. ${ }^{21}$ The ZFKFK derivative in solution (i.e. prepared using $0.6 \mathrm{mM}$ and $6 \mathrm{mM}$ of ZFKFK) presents a negative band centred at around $239 \mathrm{~nm}$, which is related to the aromatic side-groups, and a positive band at around $224 \mathrm{~nm}$ (see Fig. 2a). In the gel state (15 mM), 

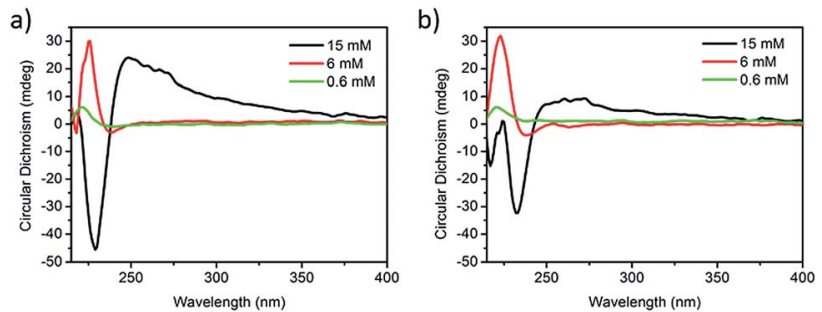

Fig. 2 Circular dichroism spectra of (a) pure ZFKFK and (b) ZFKFK : PPV at different concentrations of ZFKFK (at $20{ }^{\circ} \mathrm{C}$ ). The concentration of PPV is fixed at $0.012 \mathrm{mM}$.

a broad positive band between 249 and $282 \mathrm{~nm}$, an intense negative band at around $229 \mathrm{~nm}$ and a small positive one at $218 \mathrm{~nm}$ appear, suggesting that the chromophores organize differently in the gel state with respect to the solution state. In the case of ZFKFK : PPV mixtures in solution, the spectra show almost identical CD bands than for the pure tetrapeptides (see Fig. 2b). Remarkably, the ZFKFK : PPV mixture in its gel state shows red-shifted $\mathrm{CD}$ bands compared to the pure peptide ( $\Delta \lambda$ of $4 \mathrm{~nm}$ and $7 \mathrm{~nm}$ for the negative and positive CD bands, respectively) (see Fig. 2), which indicates that the PPV is also playing an important role on the conformation/assembly of the peptide when it is in the gel state. Remarkably, the stronger CD signals for both pure ZFKFK and ZFKFK : PPV mixture were obtained when the ZFKFK is in its gel state (see black lines in Fig. 2), which was already observed for related systems. ${ }^{21,22}$ Note that we did not observe an induced circular dichroism in the region where the PPV absorbs (Fig. S3†), which may indicates that the PPV has no preferential chiral organization when interacting with the chiral peptide assemblies (see below).

Variable-temperature studies were performed to study the self-assembly properties of the gels. At higher temperatures, the supramolecular interactions responsible for the formation of the gel are broken, going from gel to solution state (gel-sol transition). In this view, temperature melting experiments were carried out on ZFKFK and ZFKFK : PPV mixtures, increasing the temperature from $20{ }^{\circ} \mathrm{C}$ to $65{ }^{\circ} \mathrm{C}$ at a rate of $1{ }^{\circ} \mathrm{C} \min ^{-1}$ (with a stabilization time at selected temperatures); and then decreasing the temperature at the same rate and stabilization time (see detailed protocol in ESI $\dagger$ ). The heating cycle shows that at higher temperatures the CD spectra are similar for both ZFKFK and ZFKFK: PPV mixtures, even for the samples prepared with $15 \mathrm{mM}$ ZFKFK (Fig. S4-S6†). Regarding the cooling cycle, all spectra obtained for pure peptide and mixtures, both for higher and for lower temperatures, are similar (Fig. S7-S9†). Altogether, the CD spectra indicate that different self-assembled states were obtained depending on the heating-cooling cycle employed (fast or slow cooling). More stable gel structures should be obtained using a controlled cooling procedure versus most likely metastable states upon a faster cooling. A similar behavior has been reported for related systems, for which the diverse kinetic states can be trapped notably as a consequence of changes of concentration and, pH. ${ }^{22,23}$ Importantly, for the mixture, only a metastable gel (i.e. gels formed with $15 \mathrm{mM}$ of ZFKFK using a fast cooling) produces noticeable changes in CD spectra (Fig. S10 $\dagger$ ). In this regard, the $\mathrm{CD}$ spectra show a red-shift of the negative band centered at $230 \mathrm{~nm}$ on going from the metastable to the thermostable gel (Fig. S11 and S12 $\dagger$ ). Furthermore, the intensity of the broad positive band between 249 and $282 \mathrm{~nm}$ is also lower for the stable specie. The behaviors of the different selfassembled states (metastable and thermostable gel structures) were also evidenced by fluorescence spectroscopy (Fig. S13 $\dagger$ ). The fluorescence enhancement is more important for the gel prepared using a fast cooling method (metastable gel), which may indicate that the fast aggregation of the peptide prevents polymer aggregation. Similar results are observed when the polymer solution is mixed with an already formed metastable gel at room temperature, suggesting that under fast cooling, ZFKFK self-assembles quickly into metastable fibers and afterwards interacts with the polymer (Fig. S14 and S15†).

The microscopic morphology of thin deposits of pure compounds and their mixture was studied by tapping-mode atomic force microscopy (TM-AFM) and transmission electron microscopy (TEM). Thin deposits of the pure peptide (Fig. 3a and S16 $\dagger^{\circ}$ ) showed a film with an entangled network of fibrils characterized by a width around $23 \pm 6 \mathrm{~nm}$ and a thickness ranging around $3.2 \pm 1.1 \mathrm{~nm}$. In contrast, thin deposits of the pure polymer show thin layers with a thickness around $2 \mathrm{~nm}$ (Fig. S17†). Thin deposits of the mixture (at concentrations of $1 \mathrm{mM}$ in peptide and $0.01 \mathrm{mM}$ in PPV) displayed very long (a few $\mu \mathrm{m})$ and connected fibrils (Fig. 3b). The single fibrils are not helical but can bundle into twisted fibers, as shown in the bottom of Fig. 3b and in Fig. S18. $\dagger$ The measured width and thickness of single fibrils are $35 \pm 9 \mathrm{~nm}$, and $3 \pm 1 \mathrm{~nm}$,
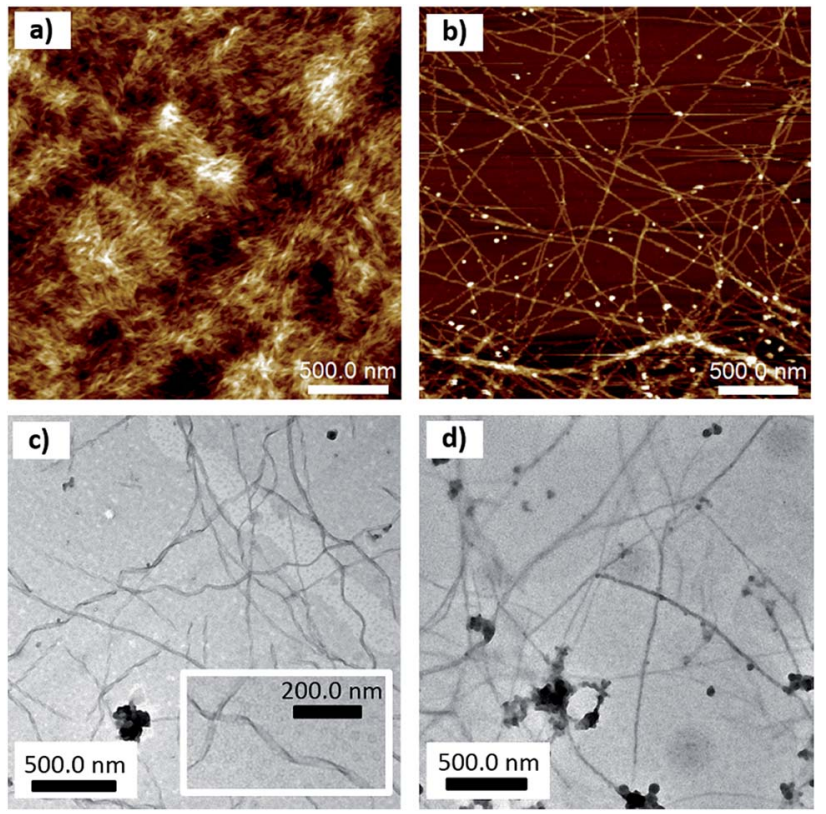

Fig. 3 TM-AFM height images of thin deposits of: (a) ZFKFK; (b) ZFKFK : PPV (concentration of $1 \mathrm{mM}$ of peptide and $0.01 \mathrm{mM} \mathrm{PPV}$ ) on mica (vertical height scale: $20 \mathrm{~nm}$ and $15 \mathrm{~nm}$, respectively). (c and d) TEM micrographs of the fibrillar network in hydrogels of (c) ZFKFK (15 $\mathrm{mM}$ ), and (d) ZFKFK : PPV (with PPV concentration of $0.012 \mathrm{mM}$ ). 
respectively. The larger width of the fibrils in deposits from the ZFKFK : PPV mixture with respect to the pure ZFKFK likely arises from the interaction of the polymer along the peptide fibrils. Likewise, TEM on gels also revealed that the presence of PPV changes the morphology of the fibrillar networks. As can be observed in Fig. 3c and $\mathrm{S} 19, \dagger$ the hydrogel of ZFKFK presents a network of twisted fibers of around $30 \mathrm{~nm}$ in width, with a right-handed helical shape, with a helical pitch ranging between 100 and $200 \mathrm{~nm}$. These fibrils are more unidirectional in the case of the ZFKFK : PPV mixture (Fig. 3d and S21 $\dagger$ ). The fact that the ZFKFK : PPV mixture shows long, unidirectional single fibrils on the surface probably comes from a difference in rigidity and/or with a lesser extent of fibril-fibril interactions when the anionic polymer is attached to the fibrils. The lack of helicity in the single fibrils when going from pure ZFKFK fibrils to ZFKFK : PPV fibrils is in line with the absence of induced CD (i.e. no CD signal in the range where PPV absorbs) in the mixture.

Taking into account the characteristics of the hybrid ZFKFK : PPV hydrogel, especially for the 'metastable' state, we were interested in its exploitation for drug delivery hydrogel, for which one could take advantage of the fluorescence properties for the localization of the drug-loaded gel. ${ }^{24}$ Indeed, it was previously shown that hydrogels can allow for a controlled release of organic dyes, which can be very useful in order to achieve multi-kinetic drug liberation..$^{2,7,13,25,26}$ To assess the use of our systems as releasing hydrogels, a solution of $25 \mu \mathrm{M}$ of methylene blue dye (MB), a model organic dye (see inset Fig. 4), was added both to the ZFKFK hydrogel (15 mM) and to the mixed ZFKFK : PPV hydrogel. After a stabilisation time of 30 minutes, $1 \mathrm{~mL}$ of supernatant aqueous solution (with Tris buffer) was added on top of the different hydrogels, and the release of $\mathrm{MB}$ was monitored from the two different gels in situ by following the absorbance at $664 \mathrm{~nm}$ (maximum absorption wavelength of free MB, Fig. S22 $\dagger$ ) of the supernatant solution (Fig. 4) each 15 minutes for 20 hours.

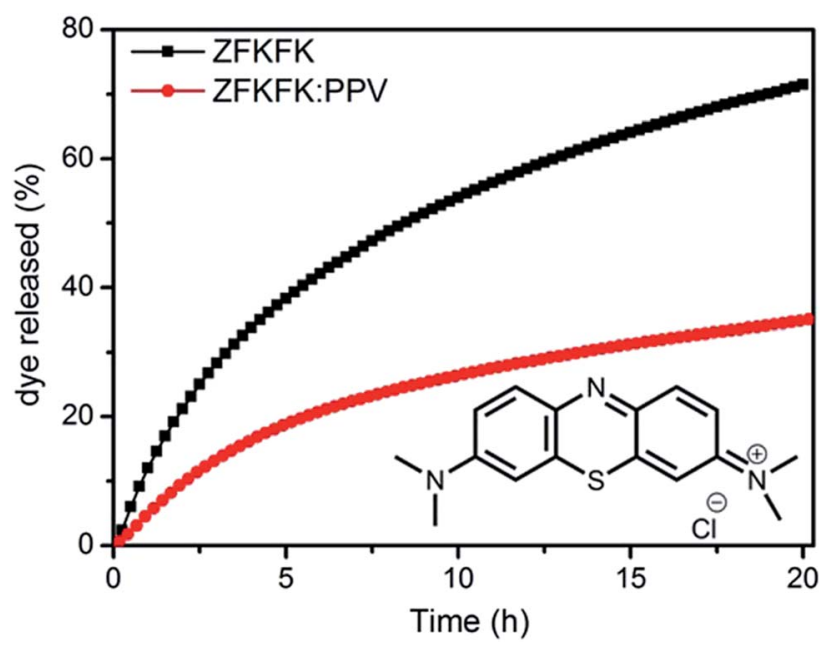

Fig. 4 Release profile of methylene blue from pure ZFKFK hydrogel (black line) and ZFKFK : PPV hydrogel (red line) after the addition of 1 $\mathrm{mL}$ of aqueous solution with Tris buffer. [ZFKFK] $=15 \mathrm{mM}$ and $[\mathrm{PPV}]=$ $0.012 \mathrm{mM}$
After 20 hours of addition of supernatant (buffered water), the release profiles obtained are clearly different for the pure ZFKFK gel and for the ZFKFK : PPV hybrid gel, showing a faster release profile for the first one (see Fig. 4). Note also that there is no evidence of PPV in the supernatant solution, as observable from UV-Vis spectra and a macroscopic view of gels (Fig. S23 and $\mathrm{S} 24 \dagger$ ). The decrease in the rate of drug release may arise by the presence of PPV at the exterior of the fibrils (as a wrapping layer) within the gel (see above). The different kinetics of release of pure ZFKFK hydrogel and ZFKFK : PPV hydrogel arises from the different hydrogel network morphology. Our hypothesis is that the favourable electrostatic interactions and $\pi$-type interactions between the polymer and the dye diminishes the extent of release compared to the pure ZFKFK gels. These interesting properties could be used for instance in multi-kinetic drug liberation applications.

\section{Conclusions}

A hybrid hydrogel was designed by combining an amphipathic low molecular weight peptide and a water-soluble $\pi$-conjugated polymer via self-assembly. Interestingly, the PPV polymer shows a higher fluorescence intensity when it is embedded in the ZFKFK hydrogel matrix than pure in solution. Accordingly, the morphology of the mixed hydrogel shows a network of longer and unidirectional fibrils compared to the helically twisted fibrils for the pure peptide. This shows that synergistic properties can arise through a supramolecular design of hybrid gels. In addition, the incorporation of PPV allows for modifying (and possibly tuning) the release of small organic dye, which could constitute an interesting fluorescence hydrogel model for releasing molecular drugs.

\section{Acknowledgements}

The authors thank the Cost Action CM 1304 (Emergence and Evolution of Complex Chemical Systems) for a Short Term Scientific Missions (STSM) of M. T.-S. in Mons. Research in Mons is supported by the Science Policy Office of the Belgian Federal Government (BELSPO - PAI 7/05). This work was also supported by the Fonds de la Recherche Scientifique - FNRS under the under the grants $n^{\circ} 1 . B 333.15 \mathrm{~F}$ (CHIRNATES) and $\mathrm{n}^{\circ} \mathrm{F} .4532 .16$ (MIS-SHERPA). J. R.-M. is FNRS post-doctoral researcher and M. S. is FNRS research associate. This work was also supported by the Ministry of Economy and Competitiveness of Spain (Grant CTQ2012-37735) and Universitat Jaume I (Grant P1-1B2013-57). M. T.-S. thanks the Ministry of Education, Culture and Sport of Spain for an FPU fellowship.

\section{Notes and references}

1 A. R. Hirst, B. Escuder, J. F. Miravet and D. K. Smith, Angew. Chem., Int. Ed., 2008, 47, 8002.

2 S. S. Babu, V. K. Praveen and A. Ajayaghosh, Chem. Rev., 2014, 114, 1973. 
3 R. V. Ulijn, N. Bibi, V. Jayawarna, P. D. Thornton, S. J. Todd, R. J. Mart, A. M. Smith and J. E. Gough, Mater. Today, 2007, 10, 40.

4 W. T. Truong, L. Lewis and P. Thordarson, Biomedical Applications of Molecular Gels, Royal Society of Chemistry, 2014.

5 R. G. Weiss, J. Am. Chem. Soc., 2014, 136, 7519.

6 D. K. Smith, in Supramolecular Chemistry: From Molecules to Nanomaterials, ed. P. A. Gale and J. W. Steed, 2012, vol. 7, p. 3355.

7 D. J. Cornwell and D. K. Smith, Mater. Horiz., 2015, 2, 279.

8 K. Hanabusa, A. Itoh, M. Kimura and H. Shirai, Chem. Lett., 1999, 767.

9 L. E. Buerkle and S. J. Rowan, Chem. Soc. Rev., 2012, 41, 6089.

10 J. R. Moffat, G. J. Seeley, J. T. Carter, A. Burgess and D. K. Smith, Chem. Commun., 2008, 4601.

11 D. J. Cornwell, B. O. Okesola and D. K. Smith, Soft Matter, 2013, 9, 8730.

12 P. D. Thornton, R. J. Mart, S. J. Webb and R. V. Ulijn, Soft Matter, 2008, 4, 821.

13 M. Tena-Solsona, S. Alonso-de Castro, J. F. Miravet and B. Escuder, J. Mater. Chem. B, 2014, 2, 6192.

14 X. Feng, L. Liu, S. Wang and D. Zhu, Chem. Soc. Rev., 2010, 39, 2411.
15 H. Jiang, P. Taranekar, J. R. Reynolds and K. S. Schanze, Angew. Chem., Int. Ed., 2009, 48, 4300.

16 C. Zhu, L. Liu, Q. Yang, F. Lv and S. Wang, Chem. Rev., 2012, $112,4687$.

17 Z. Gu, Y.-J. Bao, Y. Zhang, M. Wang and Q.-D. Shen, Macromolecules, 2006, 39, 3125.

18 C. Hao, L. Xu, W. Ma, X. Wu, L. Wang, H. Kuang and C. Xu, Adv. Funct. Mater., 2015, 25, 5816.

19 X. Wu, L. Xu, W. Ma, L. Liu, H. Kuang, W. Yan, L. Wang and C. Xu, Adv. Funct. Mater., 2015, 25, 850.

20 X. Wu, L. Xu, W. Ma, L. Liu, H. Kuang, N. A. Kotov and C. Xu, Adv. Mater., 2016, 28, 5907.

21 M. Tena-Solsona, J. F. Miravet and B. Escuder, Chem.-Eur. J., 2014, 20, 1023.

22 S. Diaz-Oltra, C. Berdugo, J. F. Miravet and B. Escuder, New J. Chem., 2015, 39, 3785.

23 M. Tena-Solsona, B. Escuder, J. F. Miravet, V. Casttelleto, I. W. Hamley and A. Dehsorkhi, Chem. Mater., 2015, 27, 3358.

24 H. Shigemitsu and I. Hamachi, Chem.-Asian J., 2015, 10, 2026.

25 K. J. Skilling, F. Citossi, T. D. Bradshaw, M. Ashford, B. Kellam and M. Marlow, Soft Matter, 2014, 10, 237.

26 D. J. Cornwell, B. O. Okesola and D. K. Smith, Angew. Chem., Int. Ed., 2014, 53, 12461. 